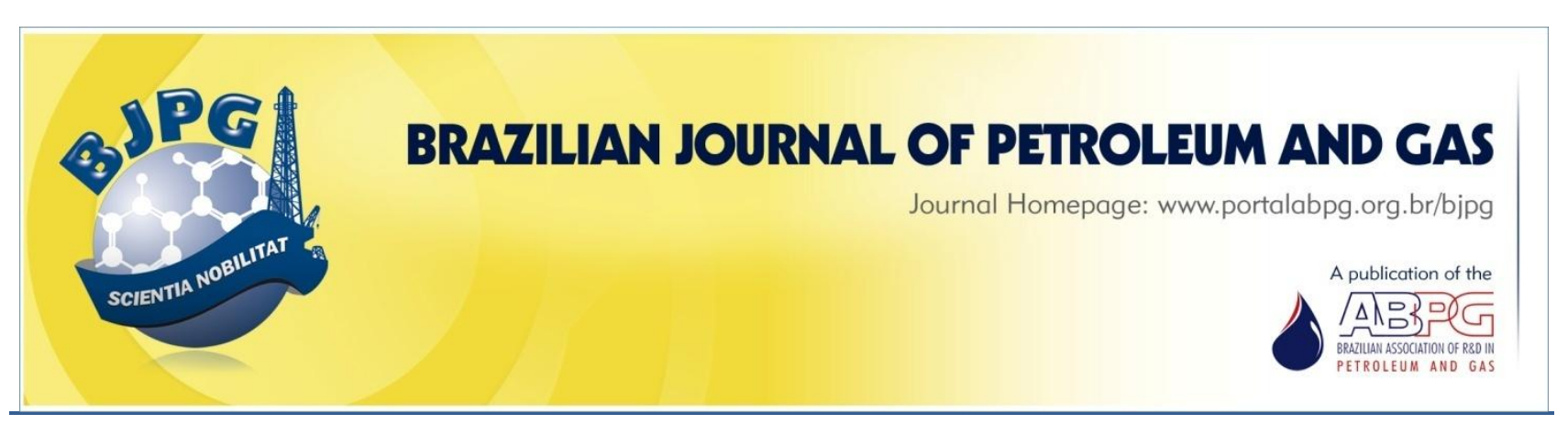

\title{
DEVELOPMENT OF A COMPUTATIONAL TOOL FOR ESP SYSTEM SIZING
}

\author{
${ }^{a}$ Oliva, G. B. F. F. ${ }^{1}$; ${ }^{a}$ Galvão, H. L. C.; ${ }^{a}$ Santos, D. P.; ${ }^{a}$ Diniz, A. A. R.; ${ }^{a}$ Maitelli, A. L.; \\ ${ }^{a}$ Costa, R. O.; ${ }^{a}$ Maitelli, C. W. S. P. \\ ${ }^{a}$ Centro de Ciências Exatas e da Terra, Centro de Tecnologia, Universidade Federal do Rio Grande do Norte, Natal-RN, Brasil \\ Received: 25.02.2016 / Revised: 09.05.2016 / Accepted: 21.09.2016 / Published on line: 04.10.2016
}

\section{ABSTRACT}

This paper presents a computational sizing tool for artificial lift by Electric Submersible Pump (ESP), considering well mechanical scheme, reservoir, fluid properties, and production data as input. This tool provides data to the user about pump types, motor, seal, cables, and auxiliary equipment for proper performance. The developed program exhibits graphs and alarms, which may help the user, while sizing the system. Also, it contains an extensive database with information about equipment used in the petroleum industry. Results from this developed tool were compared with those from a commercial program used in the industry, obtaining coherent and satisfactory results.

\section{KEYWORDS}

artificial lift; electric submersible pump; system sizing; modeling; software

\footnotetext{
${ }^{1}$ To whom all correspondence should be addressed.

Address: Universidade Federal do Rio Grande do Norte - UFRN, Centro de Ciências Exatas e da Terra - CCET, Centro de Tecnologia, CT. - Caixa Postal 1524, Campus Universitário Lagoa Nova, Natal/RN, Brasil ZIP Code: 59078-970 | Telephone: +55 (84) 99611-1000 |e-mail: gabrielbessafreitas@hotmail.com doi:10.5419/bjpg2016-0014
} 


\section{INTRODUCTION}

The artificial lift method by ESP provides onshore and offshore applicability because of its standout performance on high flow rates of liquid and high Basic Sediment and Water (BSW) wells. It is composed of surface and subsurface equipment. The surface equipment comprises a switchboard, transformers, a wellhead, and an electrical power supply. On the subsurface, the main components are: electric motor, protector or seal section, pump, and cables that are inside the well, sheltered by the casing, as shown in Figure 1 .

In the ESP system, electric power is transmitted to the bottom of the well through an electrical cable. Then, it is converted to mechanical energy by a subsurface motor that is connected to a centrifugal pump. The pump transmits energy to the fluid in the form of pressure, lifting it up to the surface.

From this context, the objective of this work is to develop a computational tool, the ESP Sizing
Software, with a similar performance to tools available on the market, at a low cost, easy to handle and maintain. This work will also compare its results to those obtained from a commercial program used in the petroleum industry.

\section{THEORETICAL ASPECTS}

In this section, the main theoretical topics for developing this work, as well as the key equipment of the ESP system are explained.

\subsection{Reservoir performance}

Two methods were used in this work to represent the Inflow Performance Relationships (IPR) curves. One of them was the linear IPR method, which accounts for liquid and incompressible single-phase. The second one was the Vogel's IPR correlation when there is multiphase oil and gas flow (Brown\& Beggs, 1977).

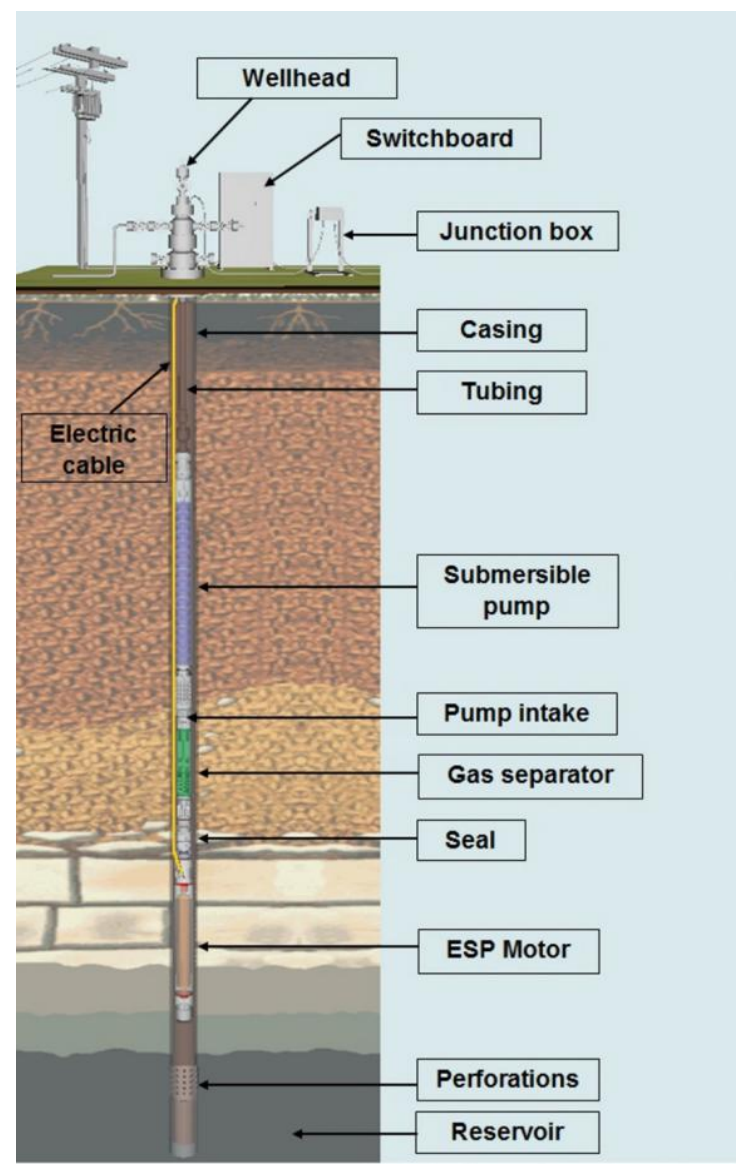

Figure 1. Equipment of Electric Submersible Pump system. 


\subsection{Centrifugal pump analysis}

From the centrifugal pump performance curves, operational range and total dynamic head are obtained. This performance is defined for a given speed with a head versus flow rate curve. One can control the speed and hydraulic output of the pump by varying the power supply frequency because changes of the motor's operating frequency impact pump speed proportionally. When the pump speed changes, head, power, and flow rate change accordingly, resulting in new curves. To understand this concept, one can consider a frequency $f_{1}$, a flow rate $Q_{1}$, a head $H_{1}$, and a power Pow ${ }_{1}$. Changing $f_{1}$ to a different value of $f_{2}$ affects $Q_{1}, H_{1}$, and Pow ${ }_{1}$, with no effect in efficiency, as expressed by Eq. (1), (2), and (3).

$Q_{2}=Q_{1}\left(\frac{f_{2}}{f_{1}}\right)$

$H_{2}=H_{1}\left(\frac{f_{2}}{f_{1}}\right)^{2}$

$\operatorname{Pow}_{2}=\operatorname{Pow}_{1}\left(\frac{f_{2}}{f_{1}}\right)^{3}$

Where $Q_{2}, H_{2}$, and $P_{0} w_{2}$ are the new values after changing $f_{1}$. Figures 2 and 3 illustrates the pump curves behavior with affinity laws.

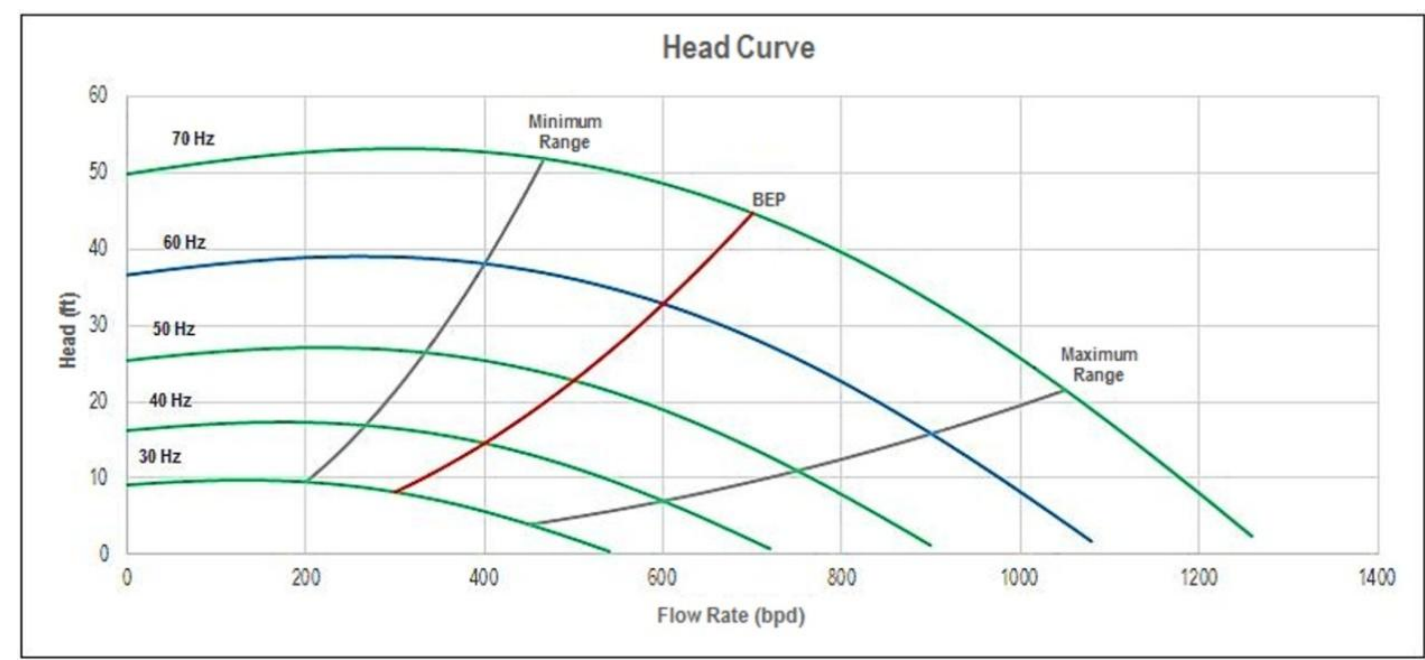

Figure 2. Performance curves of the head.

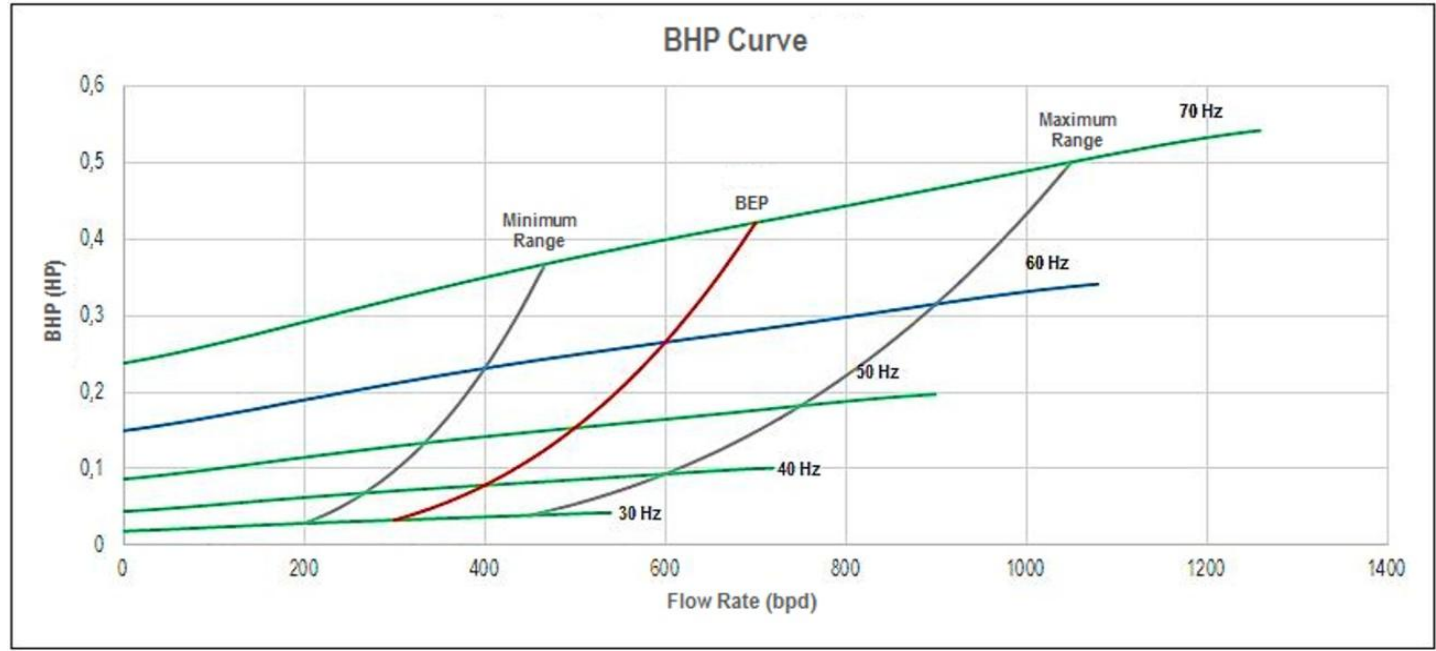

Figure 3. Performance curves of the BHP. 


\subsection{Adjustment for viscosity and free gas effects}

Increased fluid viscosity may lead to decreased head, decreased efficiency, and increased power required by the pump. With no adjustment, the system may overload or not supply enough energy to reach the desired flow rate. One approach used in the developed software to solve this problem is the model proposed by Turzo et al. (2000). The adjustment is made with four flow-rate points, with adjustment factors for flow rate, head, and efficiency. The new head curve is plotted with two more points in addition to the other four points mentioned previously (Prado, 2007). In the ESP Sizing Software, new polynomials generated by
Newton's divided differences interpolation represent the adjusted curves through viscosity effect of the fluid over the pump. Head, brake horsepower (BHP), and efficiency curves considering this effect were depicted in Figures 4, 5 , and 6 , respectively.

Pump head reduction behaves according to the quantity of free gas within the pump, and there are two regions where this decrease happens distinctively (Takács, 2008). One of them is over the stable point of best energetic efficiency, and the second one includes the unstable region under $B E P$. To calculate the head regarding gas effect and stable region, it was considered as a homogeneous model that accounts for the behavior of a

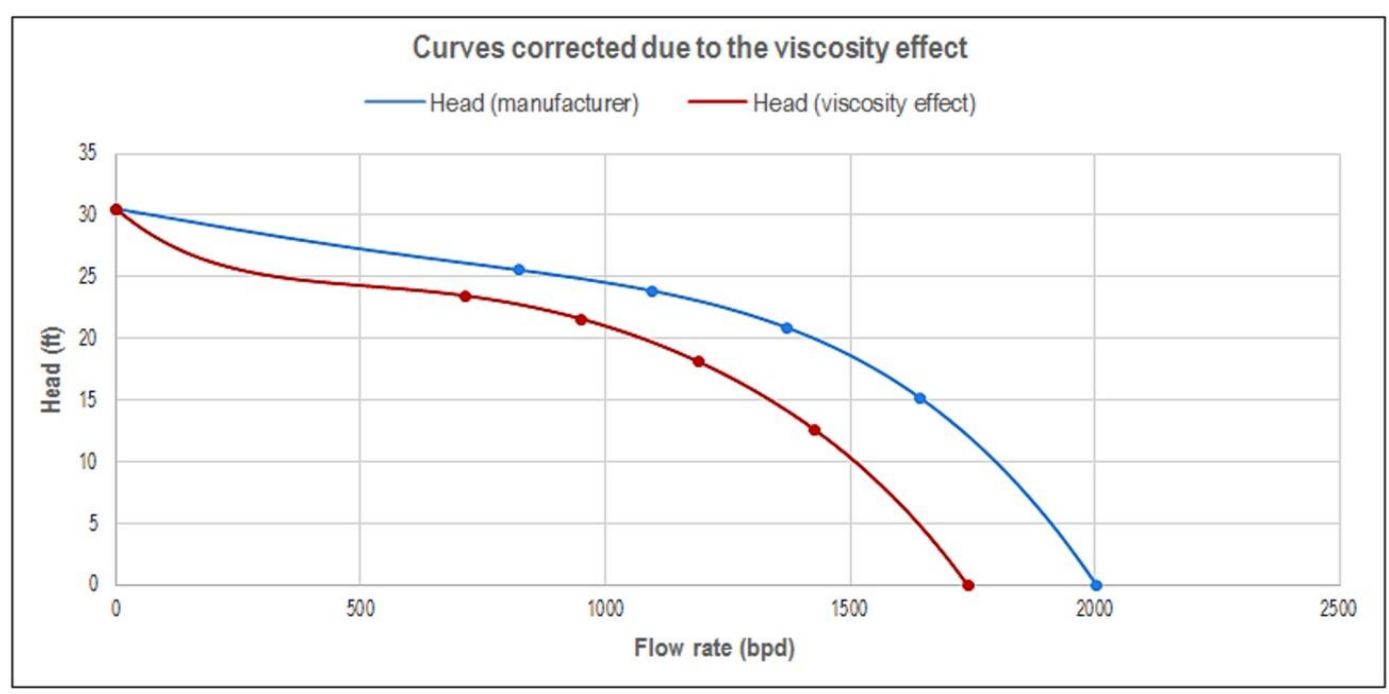

Figure 4. Head curves with correction considering the viscosity effect.

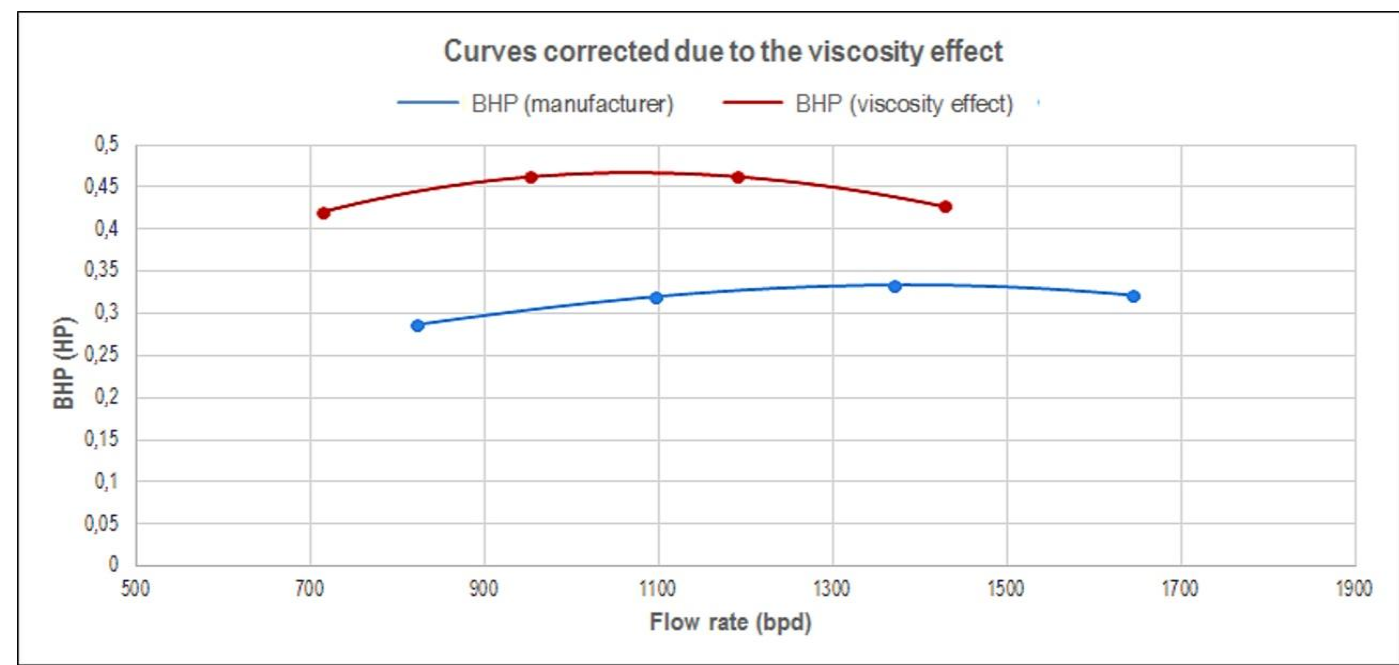

Figure 5. BHP curves with correction considering the viscosity effect. 


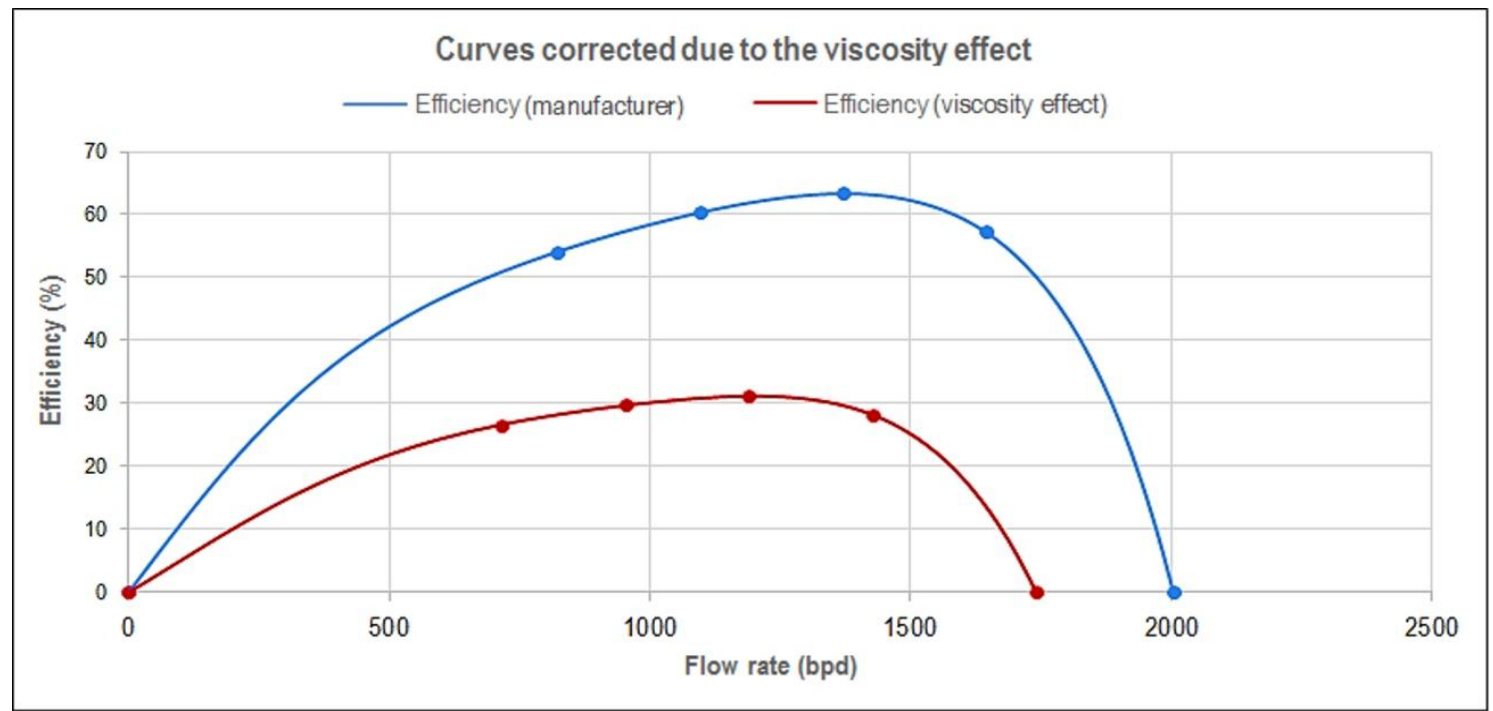

Figure 6. Efficiency curves with correction considering the viscosity effect.

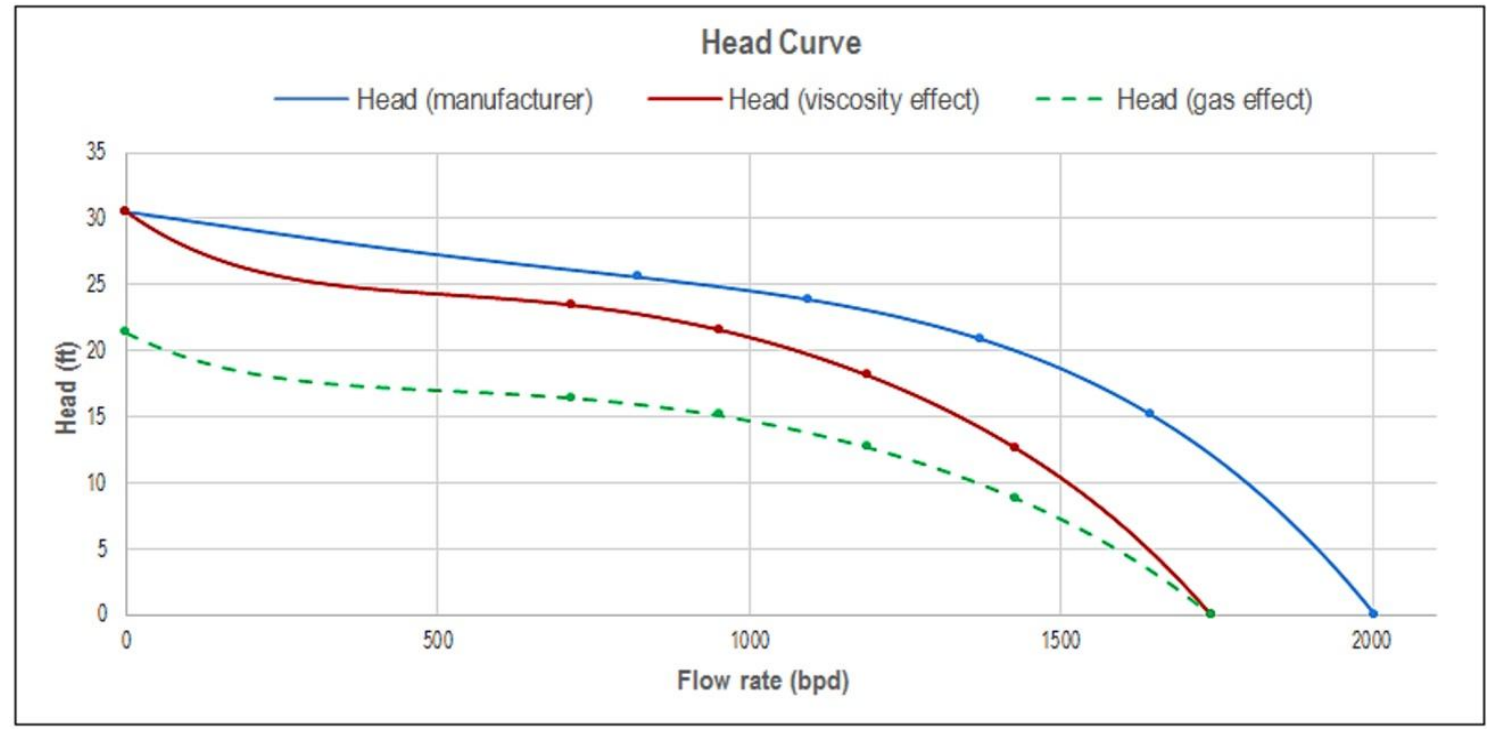

Figure 7. Head curves with correction considering viscosity effect and free gas in the pump.

homogeneous mixture while being pumped (Prado, 2007), according to Eq. (4). Head curve considering gas effect is shown in Figure 7.

$H_{g}=H_{v} \cdot\left(\frac{\rho_{m}}{\rho_{l i q}}\right)$

Where $H_{g}$ is head considering gas effect $(m), H_{v}$ is head considering viscosity effect, $\rho_{\mathrm{m}}$ is the density of the mixture $\left(\mathrm{kg} / \mathrm{m}^{3}\right)$, and $\rho_{\text {liq }}$ is the density of the liquid phase.

\subsection{Determination of Total Dynamic Head (TDH)}

Head increase by the pump impacts directly the total number of stages and other equipment choices. Two mathematical modeling were implemented to determine the head. The first one was designed considering liquid single-phase flow, and the second one considering multiphase flow. The total dynamic head considering single-phase flow was defined by Eq. (5). 


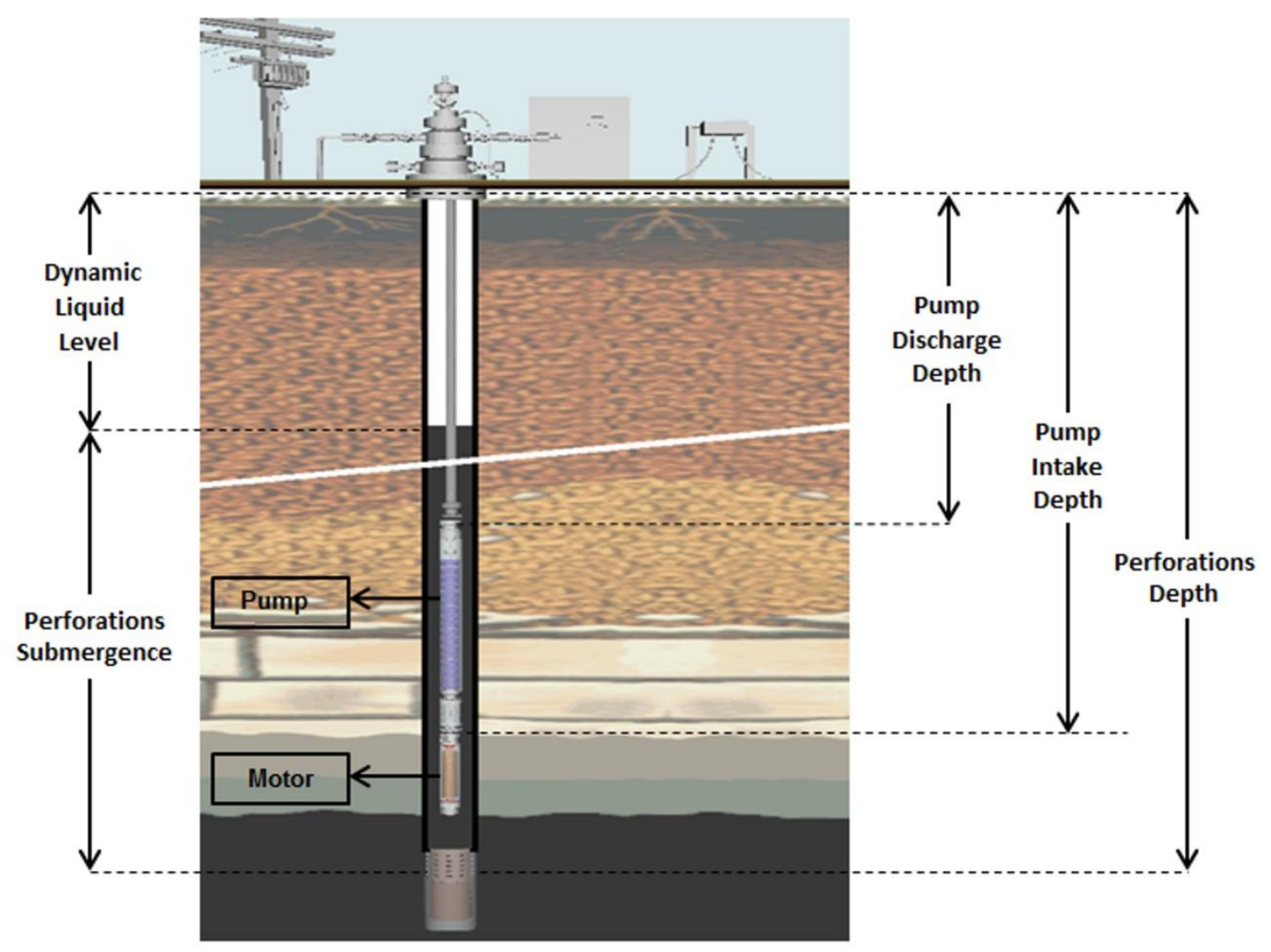

Figure 8. Parameters of the system.

$T D H=D L+\Delta P_{f r c}+\frac{\left(P_{h d}-P_{c a s}\right)}{0.1 \cdot \gamma_{l i q}}$

Where $P_{\text {hd }}$ is head pressure $\left(\mathrm{kgf} / \mathrm{cm}^{2}\right), P_{\text {cas }}$ is casing pressure, and $\gamma_{\text {liq }}$ is relative density of liquid (dimensionless). $\Delta \mathrm{P}_{\text {fric }}$ and $\mathrm{DL}$ were obtained by $\mathrm{Eq}$. (6) and (7), respectively.

$$
\begin{aligned}
& \Delta P_{f r c}=\left[\frac{\left(\frac{0.000189 \cdot Q_{d}}{I D_{t u b}{ }^{2}}\right)}{1.32 \cdot C \cdot\left(\frac{I D_{t u b}}{48}\right)^{0,63}}\right]^{\frac{1}{0.54}} \cdot\left(\frac{L_{p m p}}{0.3048}\right) \\
& D L=L_{p e r}-\frac{P_{p w f}}{0.1 \cdot \gamma_{l i q}}
\end{aligned}
$$

$\Delta \mathrm{P}_{\text {fric }}$ losses caused by friction across the tubing (m) were determined through the Hazen-Williams equation (Williams \& Hazen, 1920). DL is the dynamic liquid level $(\mathrm{m})$, defined as the vertical distance from the surface to the top of the existing oil in the tubing-casing annulus. This concept was illustrated in Figure 8. $\mathrm{Q}_{d}$ is the pump desired operational flow rate $\left(\mathrm{m}^{3} / \mathrm{d}\right), I_{\text {tub }}$ is the tubing inner diameter (in), $L_{p m p}$ is the pump depth (m), $L_{\text {per }}$ is the perforation depth $(\mathrm{m})$, and $P_{p w f}$ is the pressure in front of the perforations $\left(\mathrm{kgf} / \mathrm{cm}^{2}\right)$.

When multiphase flow was considered, its correlations, characteristics of production fluids, well mechanical schemes, and operational conditions were studied to determine the total dynamic head. In this case, the TDH was expressed through Eq. (8).

$$
T D H=\frac{\Delta P_{p m p}}{0.1 \cdot \gamma_{l i q}}=\frac{\left(P_{d i s}-P_{s u c}\right)}{0.1 \cdot \gamma_{l i q}}
$$

Where $P_{\text {dis }}$ is the pump discharge pressure $\left(\mathrm{kgf} / \mathrm{cm}^{2}\right)$ and $P_{\text {suc, }}$ is the intake pressure obtained by:

$$
P_{s u c}=P_{p w f}-0.1 \cdot\left(L_{p e r}-L_{p m p}\right) \cdot \gamma_{l i q}
$$




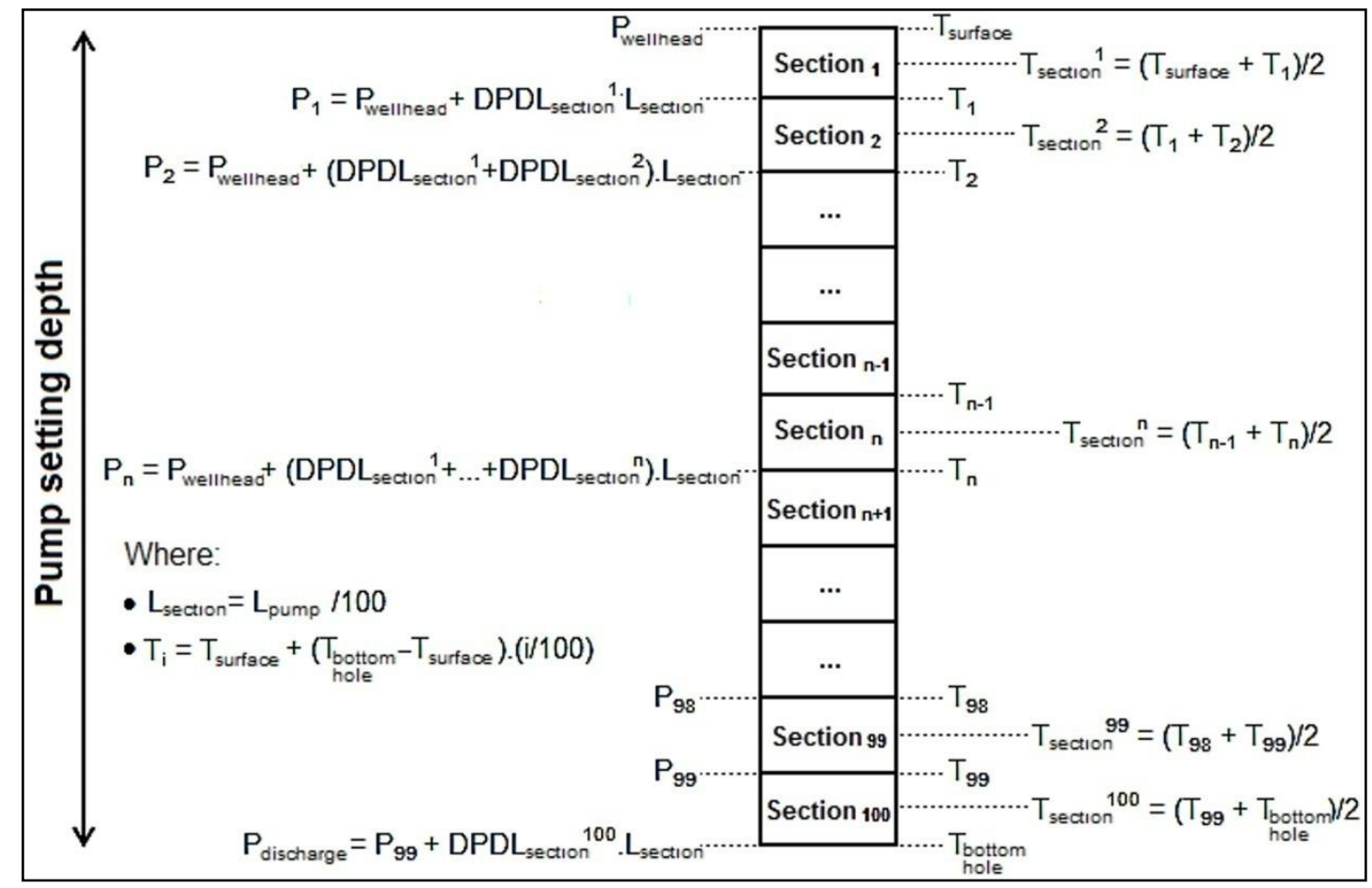

Figure 9. Simplified representation used for calculations in multiphase flow.

To obtain the discharge pressure, the developed program divided the tubing into one hundred sections of equal length. For each of them, parameters were calculated based on the multiphase flow correlations previously chosen. Based on this, the pump discharge pressure was determined conforming Eq. (10).

$$
P_{d i s}=P_{h d}+\sum_{i=1}^{100} D P D L_{\mathrm{sec}}{ }^{i} \cdot\left(\frac{L_{p m p}}{100}\right)
$$

Where $D P D L_{\text {sec }}{ }^{i}$ is the pressure gradient in section $\mathrm{i}$. The proceedings used in the program to determine pressure of the sections were portrayed in Figure 9, in a simplified way.

\subsection{Determination of the number of pump stages}

Total number of stages, $n^{\circ}{ }_{s t g}$, was defined by Eq. (11), with no adjustments.

$$
n_{\text {stg }}^{\circ}=\frac{T D H}{\text { Head/Stage }}
$$

When considered, adjustments were made individually for the head of each stage. The total number of stages was determined iteratively until the discharge pressure of a given stage surpassed system's TDH. For each stage calculations, Eq. (12) was applied to find the discharge pressure. These proceedings were represented in Figure 10.

$P_{d i s}^{k}=\left(\frac{P_{s u c}{ }^{p m p}}{14.223}\right)+\sum_{k=1}^{k}\left(\frac{\gamma_{l i q} \cdot H_{s t g}{ }^{k}}{10.01}\right)$

Where $\mathrm{H}_{\mathrm{stg}}{ }^{\mathrm{k}}$ is head for a given stage $\mathrm{k}$ and the relative density of liquid is obtained from:

$\gamma_{l i q}=\gamma_{w} \cdot B S W+\gamma_{o} \cdot(1-B S W)$

Where $\gamma_{w}$ is relative density of the water; $\nu_{0}$, of oil. BSW is the well's water percentage.

\subsection{ESP motor analysis}

According to Takács (2008), when the fluid speed around the motor is greater than $1 \mathrm{ft} / \mathrm{s}$, it is considered that the motor is well cooled down. This speed was calculated through Eq. (14). 


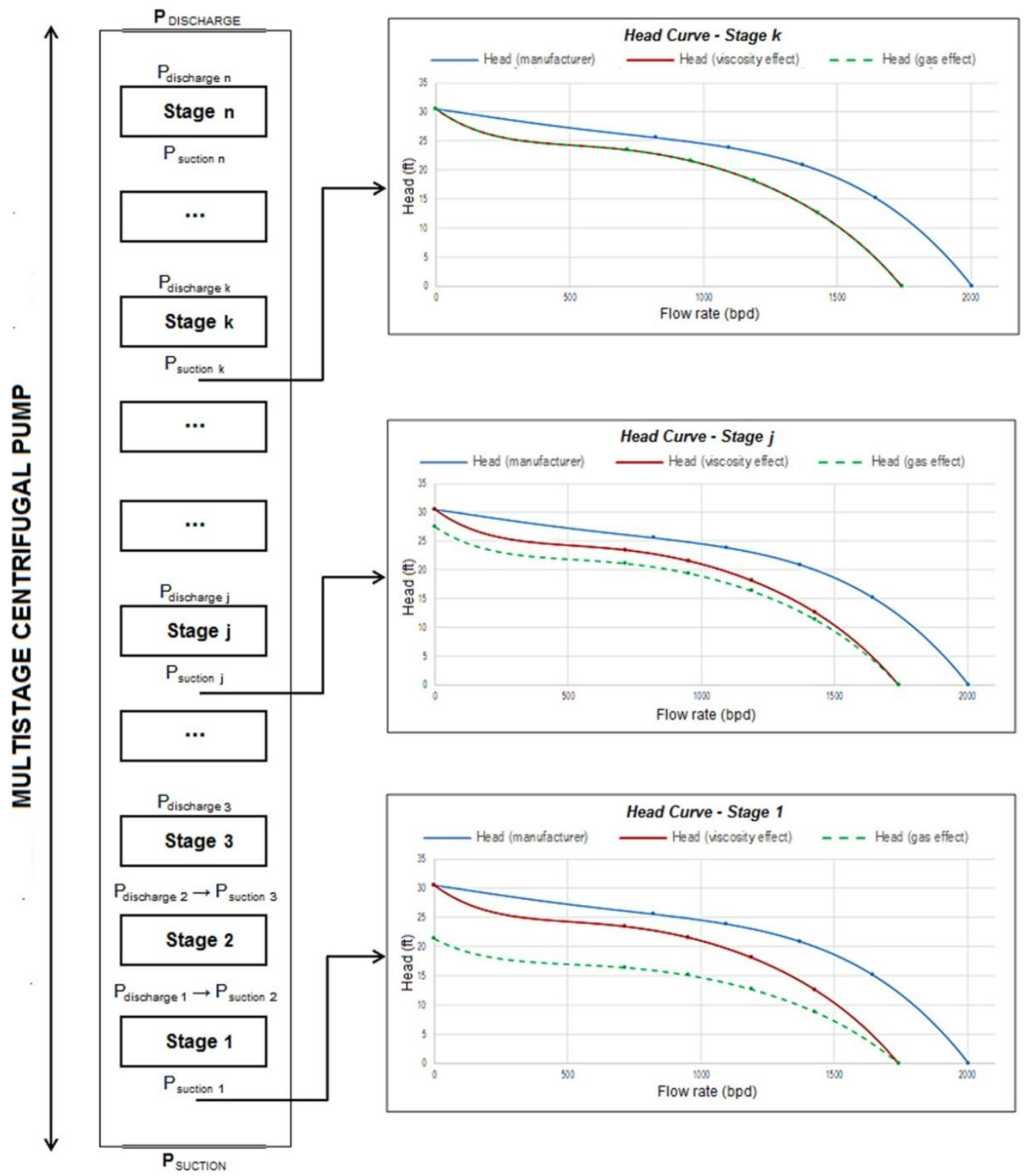

Figure 10. Simplified representation of the proceedings used in stage to calculate the ESP pump for determining the total number of stages.

$$
V=1.19 \cdot 10^{-2} \cdot \frac{q_{b p d}}{I D_{c a s}^{2}-O D_{m o t}^{2}}
$$

Where $q_{b p d}$ is the production flow rate $\left(\mathrm{m}^{3} / \mathrm{d}\right), I_{\text {cas }}$ is the casing inner diameter (in), and $\mathrm{OD}_{\text {mot }}$ is the motor's outer diameter (in). When shroud is used, $I D_{\text {cas }}$ changes to the ID of the shroud.

\subsection{Cables analysis}

Voltage drop along the cables is expected to not exceed 30 volts for every 1000 feet of cable length. To find this value, the cable resistance was determined with Eq. (15), proposed by French (1991).

$$
R_{T}=\frac{L_{c} \cdot r_{c}}{1000} \cdot\left[1+0.00214 \cdot\left(T_{c}-77\right)\right]
$$

Where $R_{T}$ is resistance for a given temperature $(\Omega)$, $L_{c}$ is cable length $(m), r_{c}$ resistance at every 1000 feet of cable at $77^{\circ} \mathrm{F}(\Omega / 1000 \mathrm{ft})$, and $T_{c}$ is cable temperature $\left({ }^{\circ} \mathrm{C}\right)$. The value of voltage drop for every 1000 feet, $U_{c}$, was estimated using the Ohm's law, so that the previous expression was rearranged to Eq. (16). 


$$
U_{c}=\frac{L_{c} \cdot r_{c}}{1000} \cdot\left[1+0.00214 \cdot\left(T_{c}-77\right)\right] \cdot I
$$

\section{ESP SIZING SOFTWARE}

The ESP Sizing Software was developed with Microsoft Office Excel platform, using Visual Basic for Applications (VBA) programming language. This program has a database containing information regarding many ESP system equipment used in the industry. Calculation procedure accounts for physical properties of multiphase flow and empirical correlations, which determine the main parameters concerning this flow type.

This software allows the user to configure characteristics of the well, reservoir, flow properties, and operating conditions to perform proper sizing of the system. The interface functionalities permit equipment filtering, warning the user of unwanted conditions, and improving the perception of what is being sized.

\subsection{Graphical User Interface}

The first screen is used for selecting the casing and the tubing, determine perforations and pump setting depth, tubing absolute roughness, well inclination, well fluid properties-degree API, BSW, GOR-specific gravity of water and gas, geothermal gradient, and surface and bottom-hole temperature data.

There is a screen focused in the well IPR curve, with mathematical modeling chosen by the user. It is possible to find the maximum production rate of a reservoir and choose a desired flow rate. To achieve its purpose, well tests (test rate and pressure), reservoir static pressure, head, and casing pressure data need to be provided by the user. Following this screen, it is possible to see the pump discharge, the pump intake, and the well flow pressures conditions to determine the pump differential pressure, which is supplied for a desired flow rate.

There is a screen for the centrifugal pump, in which it is possible to choose the pump, determine its operating frequency, and to see the liquid viscosity at the pump suction and the desired production rate. The user may use buttons to filter which pumps are in the desired operational range. Then, it is calculated the number of necessary stages and the brake horsepower, considering or not viscosity and/or gas effects. To enable stage-tostage calculation, the user may select an iterative calculation option. Head curve for a given stage was illustrated in Figure 11.

Regarding the motor, it is shown the minimum power to prevent the motor from overloading. The user may choose one of the registered motors, its

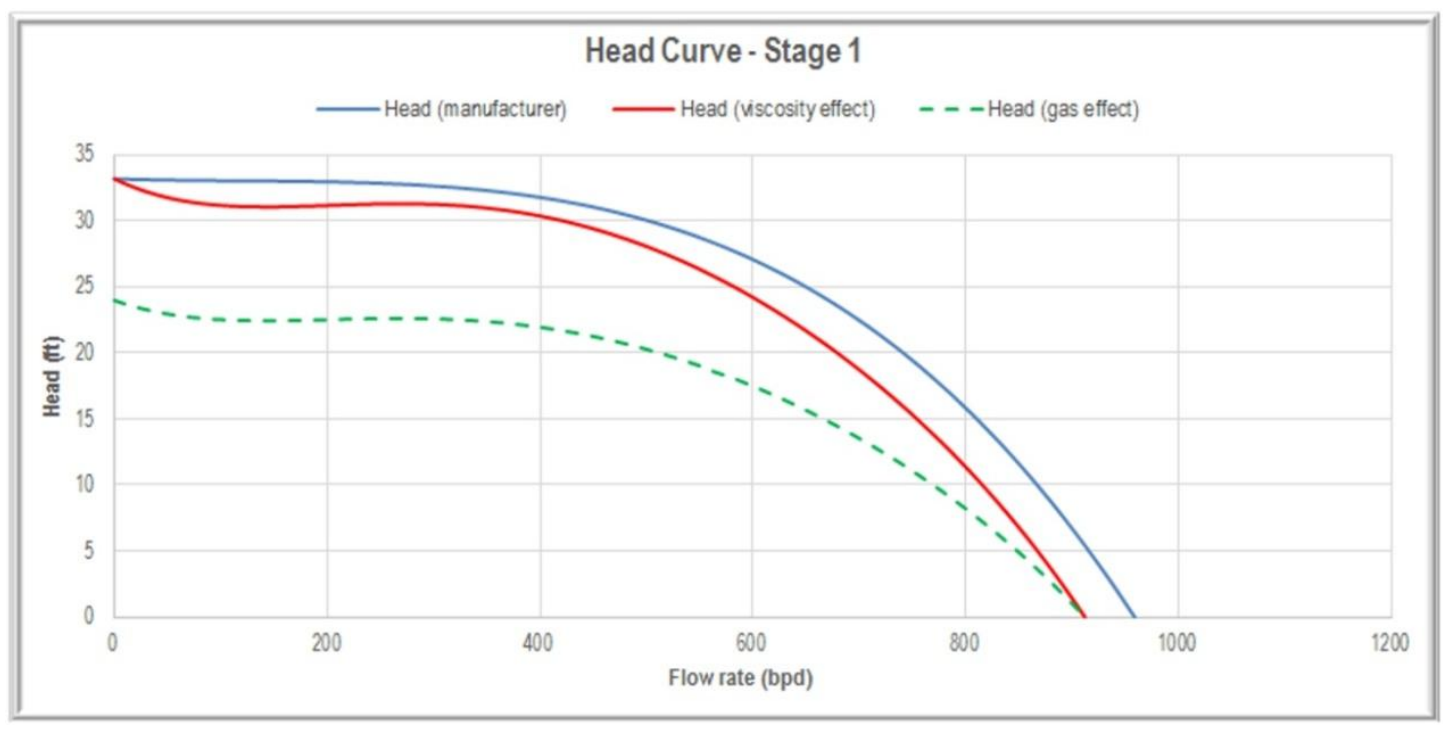

Figure 11. Performance curves of the head in a given stage. 


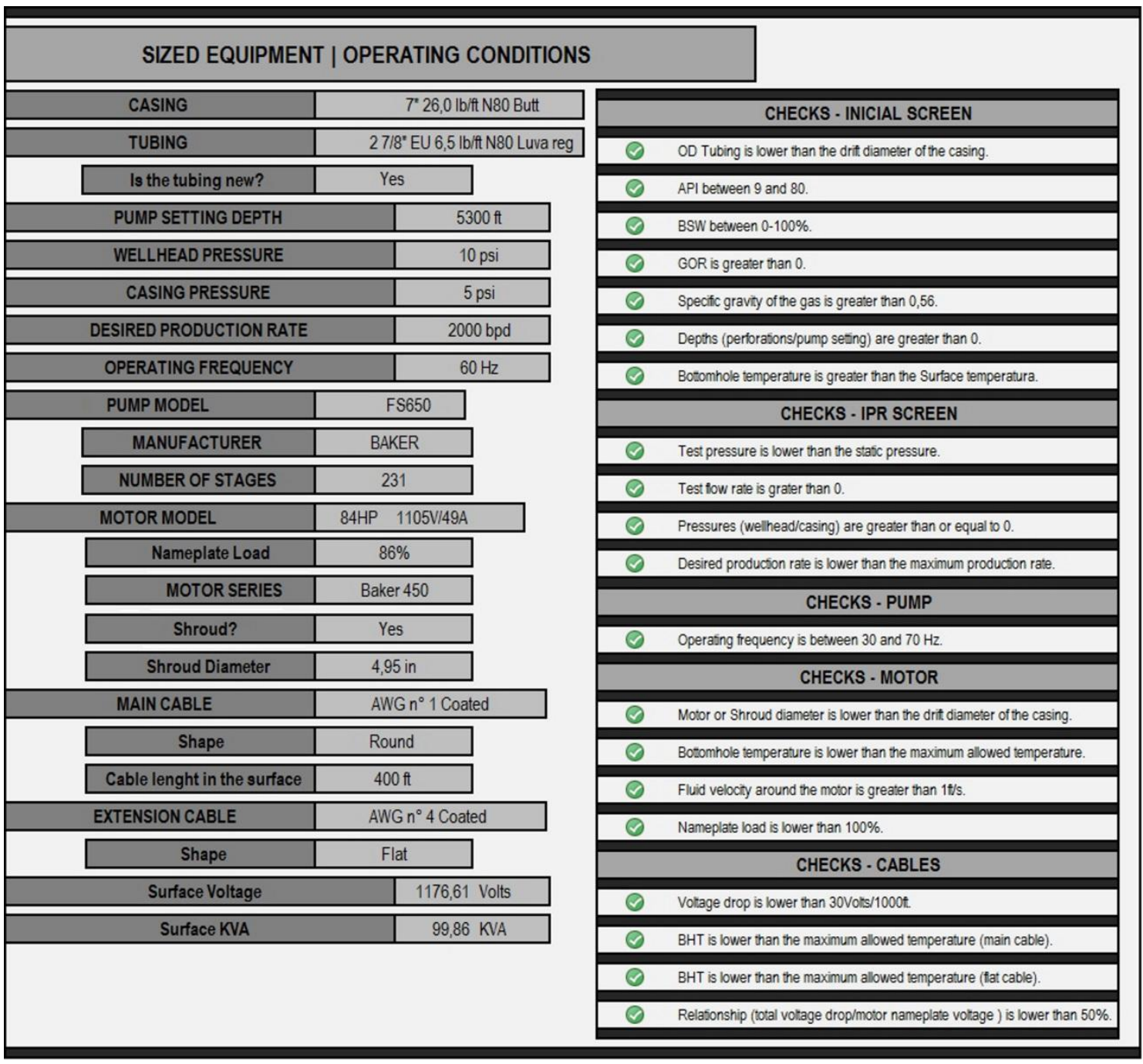

Figure 12. Screen for output.

diameter and its need of shroud. For cables sizing, the user may set the length of the cable used on the surface, choose the power cable and add information about the extension cable of the motor. The last part of the developed software is the output screen. It presents the chosen equipment during sizing, as well as some operating conditions. Each part of the graphical interface, previously mentioned, has its according verifications that were displayed in the output screen in Figure 12.

\section{RESULTS AND DISCUSSIONS}

To validate this sizing software, its results were compared with those obtained from a commercial program used in the industry and with a bibliographic example from the ESP manual. Benchmarking was done with vertical wells and the following variables: TDH, pump intake and discharge pressure, number of stages, pump brake horsepower, fluid velocity around the motor, and voltage drop across the cable. The program was also tested with another example from the bibliography when GOR was considerable.

The input parameters of the sized system were presented in Table 1 . Well 1 data was used to compare the developed program with a commercial one. In the bibliographic example comparison, Well 2 data was used. Well 3 data was important to test the developed program in a different scenario, when GOR was not null. The error variables were obtained from Eq. (17). 
Table 1. Input parameters for comparisons: Well 1 data is used for the comparison with a commercial program; Well 2 data is used for the comparison with a bibliographic reference; and Well 3 data is used for testing the gas effect consideration.

\begin{tabular}{|c|c|c|c|}
\hline \multirow{2}{*}{ Parameter } & \multicolumn{3}{|c|}{ Value } \\
\hline & Well 1 & Well 2 & Well 3 \\
\hline Casing inside diameter ( $p o l)$ & 6.366 & 6.366 & 6.331 \\
\hline Tubing inside diameter ( $\mathrm{pol}$ ) & 2.992 & 2.992 & 2.441 \\
\hline Perforations depth $(m)$ & 824 & $1,630.6$ & $1,981.1$ \\
\hline Pump setting depth $(m)$ & 730 & $1,584.9$ & $1,523.9$ \\
\hline Static pressure $\left(\mathrm{kgf} / \mathrm{cm}^{2}\right)$ & 70 & 116 & 154.7 \\
\hline Test flow rate $\left(m^{3} / d\right)$ & 220 & 143.1 & 318 \\
\hline Test pressure $\left(\mathrm{kgf} / \mathrm{cm}^{2}\right)$ & 43 & 69.3 & 61 \\
\hline Wellhead pressure $\left(\mathrm{kgf} / \mathrm{cm}^{2}\right)$ & 4 & 10.5 & 7 \\
\hline Casing pressure $\left(\mathrm{kgf} / \mathrm{cm}^{2}\right)$ & 0.07 & 0 & 1.4 \\
\hline Surface temperature $\left({ }^{\circ} \mathrm{C}\right)$ & 37.38 & 23.9 & 21.1 \\
\hline Bottom-hole temperature $\left({ }^{\circ} \mathrm{C}\right)$ & 65.56 & 82.2 & 65.6 \\
\hline Degree API & 13 & 30 & 33 \\
\hline BSW (\%) & 89 & 90 & 50 \\
\hline $\operatorname{GOR}\left(m^{3} / m^{3}\right)$ & 0 & 0 & 53.4 \\
\hline Pump model & P23 & GC2200 & GC2200 \\
\hline Pump operating frequency $(\mathrm{Hz})$ & 55.2 & 60 & 60 \\
\hline Desired flow rate $\left(m^{3} / d\right)$ & 390 & 318 & 318 \\
\hline Motor nameplate power $(H P)$ & 115 & 130 & 108 \\
\hline Motor nameplate voltage $(V)$ & 1,390 & 2,145 & 1,209 \\
\hline Motor nameplate current $(A)$ & 53 & 35 & 60 \\
\hline Power cable model & $\begin{array}{c}\text { AWG } n^{\circ} 2 \\
\text { coated }\end{array}$ & $\begin{array}{c}\text { AWG } n^{\circ} 4 \\
\text { coated }\end{array}$ & $\begin{array}{c}\text { AWG } n^{\circ} 2 \\
\text { coated }\end{array}$ \\
\hline
\end{tabular}

$\varepsilon=100 \cdot\left|\left(\frac{\text { Value }_{\text {Com }}-\text { Value }_{\text {Dev }}}{\text { Value }_{\text {Com }}}\right)\right|$

Where Value $_{\text {com }}$ stands for compared variable, from a commercial program or from the bibliographic example. Value $_{\text {Dev }}$ stands for developed variable, from the developed program.

Through analysis of Table 2, it was implied the results from the ESP Sizing Software were coherent when compared with those from a commercial program. The errors were lesser than $10 \%$ to all observable variables. It should be emphasized that the results obtained previously were achieved considering the viscosity effect. The total number of stages and the pump brake horsepower were also analyzed when this effect was not considered, so that the results were shown in Table 3.

Based on the previous results, it was noticed the importance of adjustments on the performance curves of the pump when they were exposed to different fluid conditions. For example, fluids with higher viscosities than that of water at $60^{\circ} \mathrm{C}$ and/or 
Table 2. Comparison between the results obtained from the developed program and from a commercial program.

\begin{tabular}{lccc}
\multicolumn{1}{c}{$\begin{array}{c}\text { Observed } \\
\text { Variable }\end{array}$} & $\begin{array}{c}\text { Developed } \\
\text { Program }\end{array}$ & $\begin{array}{c}\text { Commercial } \\
\text { Program }\end{array}$ & Error (\%) \\
\hline Total dynamic head $(\mathrm{m})$ & 650.94 & 663.1 & 1.83 \\
Pump intake pressure $\left(\mathrm{kgf} / \mathrm{cm}^{2}\right)$ & 12.77 & 12.92 & 1.16 \\
Pump discharge pressure $\left(\mathrm{kgf} / \mathrm{cm}^{2}\right)$ & 77.64 & 77.63 & 0.01 \\
Total number of stages & 58 & 56 & 3.57 \\
Pump brake horsepower $(\mathrm{HP})$ & 77.69 & 71.74 & 0.29 \\
Fluid velocity around the motor $(\mathrm{m} / \mathrm{s})$ & 0.996 & 0.996 & 0 \\
Voltage drop along the cable $(\mathrm{V})$ & 32.14 & 30.48 & 5.45 \\
\hline
\end{tabular}

Table 3. Comparison when the viscosity effect of the fluid over the pump was not considered.

\begin{tabular}{cccc}
\hline $\begin{array}{c}\text { Observed } \\
\text { Variable }\end{array}$ & $\begin{array}{c}\text { Developed } \\
\text { Program }\end{array}$ & $\begin{array}{c}\text { Commercial } \\
\text { Program }\end{array}$ & Error (\%) \\
\hline Total number of Stages & 52 & 56 & 7.14 \\
Pump brake horse power (HP) & 61.85 & 71.74 & 13.79 \\
\hline
\end{tabular}

Table 4. Comparing results obtained by the ESP Sizing Software and by the bibliographic reference (ESP manual).

\begin{tabular}{lccc}
\multicolumn{1}{c}{$\begin{array}{c}\text { Observed } \\
\text { Variable }\end{array}$} & $\begin{array}{c}\text { Developed } \\
\text { Program }\end{array}$ & $\begin{array}{c}\text { Commercial } \\
\text { Program }\end{array}$ & Error (\%) \\
\hline Total dynamic head $(f t)$ & $5,303.13$ & 5,461 & 2,89 \\
Pump intake pressure $(p s i a)$ & 106.92 & 106.42 & 0.47 \\
Total number of stages & 110 & 110 & 0 \\
Pump brake horse power $(H P)$ & 122.26 & 121 & 1.04 \\
Voltage drop along the cable $(V)$ & 95.32 & 109.47 & 12.93 \\
\hline
\end{tabular}

presence of free gas within the pump. In this case, the different values obtained by the developed tool when the viscosity effect was not considered may have been caused by the low oil API degree and because of the fluid viscosity at the pump intake, which was $12.8 \mathrm{cP}$.

Similar analysis was done with the ESP bibliography that performs this procedure. It was observed from Table 4 that the developed tool also showed good results for this comparison, with one error greater than $10 \%$ for voltage drop along the cable. It is worth noting that the results presented in this section were obtained without considering the viscosity effect on the pump curves, but when considered, the number of stages was increased by only one stage (111 stages).

Beside the two examples previously mentioned, sizing for the Well 3 data was done. The results 
Table 5. Results obtained by the developed program for Well 3 data to test gas effect consideration.

\begin{tabular}{lc}
\multicolumn{1}{c}{$\begin{array}{c}\text { Observed } \\
\text { Variable }\end{array}$} & $\begin{array}{c}\text { Developed } \\
\text { Program }\end{array}$ \\
\hline Total dynamic head $(\mathrm{m})$ & $1,384.8$ \\
Pump intake pressure $\left(\mathrm{kgf} / \mathrm{cm}^{2}\right)$ & 16.7 \\
Pump discharge pressure $\left(\mathrm{kgf} / \mathrm{cm}^{2}\right)$ & 150.8 \\
Total number of stages & 97 \\
Pump brake horsepower $(H P)$ & 94.63 \\
Voltage drop along the cable $(\mathrm{V})$ & 87.93 \\
\hline
\end{tabular}

were shown in Table 5. It was observed from this example that the developed program also sizes systems with GOR not null. In this case, there was no comparison because the purpose was to show that the software accounted for gas effect.

\section{CONCLUSIONS}

This paper presented an important computational tool for equipment sizing regarding the lifting system equipped with the artificial lift method by ESP. From the calculations used and optimization mechanisms implemented, it was simple to test equipment combination and choose the ones that suited better the observed system to work efficiently, safely, and inexpensively.

To validate the developed program, its results were compared to those from a commercial software and bibliographic reference from the ESP manual, achieving coherent and satisfactory results. Even with good results presented by the developed sizing software, it is important to do more benchmarking specially with systems having different characteristics from those previously presented, e.g., fluids with high viscosity and/or lower BSW values. To improve the developed program, another highlight point is to acknowledge economic factors when choosing equipment.

\section{ACKNOWLEDGEMENTS}

The authors acknowledge the PRH-PB 21, Petrobras, and researchers working at Laboratory of Automation in Petroleum (Laboratório de Automação em Petróleo - LAUT), Federal University of Rio Grande do Norte, Brazil.

\section{REFERENCES}

Brown, K.; Beggs, E. The technology of artificial lift methods. Tulsa: Kluwer Academic Publishers, 1997.

French, S .W. Optimum Cable Selection for Electrical Submersible Pump. Production Operations Symposium, Oklahoma City, Oklahoma, April, 1991. http://dx.doi.org/10.2118/21693-MS

Prado, M. G. Electrical Submersible Pumping course. Rio de Janeiro: PETROBRAS, 2007.

Takács, G. Electrical submersible pumps manual: design, operations and maintenance. USA: Gulf Professional Publishing, 2008.

Turzo, Z.; Takács, G.; Zsuga, J. A Computerized Model for Viscosity Correction of Centrifugal Pump Performance Curves. $\mathbf{4 7}^{\text {th }}$ Southwestern Petroleum Short Course, Texas, April, 2000.

Williams, G. S.; Hazen, A. Hydraulic Tables. $3^{\text {rd }}$ Edition. New York: John Wiley and Sons, 1920. 